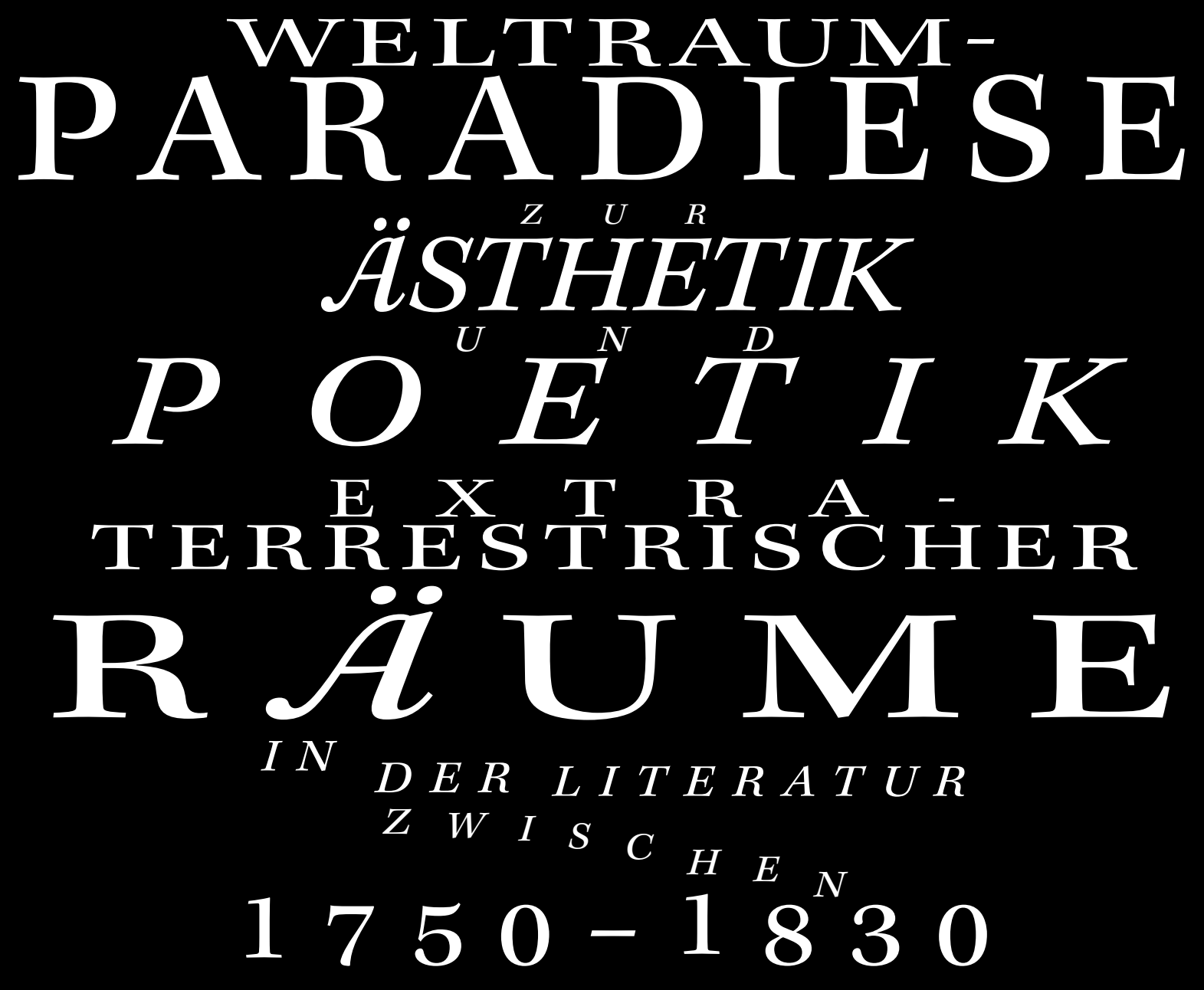


Weltraum-Paradiese. Zur Ästhetik und

Poetik extraterrestrischer Räume

in der Literatur zwischen 1750 und 1830.

$\odot$

\section{ZUR KONSTRUKTION}

\section{DES BEWOHNTEN WELTALL-SYSTEMS}

Mit dem Aufkommen des Kopernikanismus bricht eine neue Ära der Menschheit an. Die Ordnung der diabolozentrischen, aristotelisch-ptolemäischen Kosmologie scheint allmählich, aber unwiderruflich verschwunden zu sein. Das von Descartes zur philosophischen Methode entwickelte Abstraktionsdenken sowie die neuen Medien wie Teleskopie, Spektroskopie und Fotografie vertiefen unsere Kenntnisse über die Struktur des Universums und ermöglichen eine epistemische Kolonialisierung des Weltalls - eine These, die schon Camille Flammarion in Spaziergänge in der Sternenwelt (1920) formuliert, wenn er die Entwicklung der modernen Astronomie als Erfolgsgeschichte der neuen Medien preist. ${ }^{2}$ Diskutiert wird nicht nur über die auf antike atomistische Vorstellungen von Lukrez ${ }^{3}$ zurückgehende Unendlichkeit des kosmischen Raumes (etwa in Giordano Brunos De l'infinito, universo e mondi, 1584), ${ }^{4}$ sondern auch über die Strukturen des Universums, die nach 
으 Kopernikus und Galilei neu definiert werden müssen. Die philosophische Spekulation, die sich auf neueste naturwissenschaftliche Erkenntnisse und das von Immanuel Kant ${ }^{5}$ oder Emanuel Swedenborg ${ }^{6}$ thematisierte Analogiedenken stützt, lässt nicht nur eine neue Kosmographie des Sonnensystems entstehen, sondern bettet auch jene planetarische Ordnung in eine grössere, multidimensionale Systemhierarchie ein. Beispielsweise glaubt Johann Heinrich Lambert in seinen Cosmologischen Briefen (1761) an ein gemeinsames Gravitationszentrum unserer Milchstrasse, einen dunklen Himmelskörper, um den die unzähligen Subsysteme der Sonnen und ihrer Planeten kreisen. ${ }^{7}$ Aber auch in der postkopernikanischen Ära greift die epistemisch orientierte, naturwissenschaftliche Spekulation auf die altbekannten Beschreibungsmodelle zurück, die den Kosmos als einen ästhetisierten, durch die Poiesis-Allmacht eines göttlichen Künstlers erschaffenen Raum darstellen. Das gilt für Johannes Keplers Mysterium Cosmographicum (1596), ein Werk, in dem die pythagoreische und platonische Faszination für die geometrische Weltraumästhetik ${ }^{8}$ rekontextualisiert und auf die postkopernikanischen heliozentrischen Planetenstrukturen übertragen wird, genauso wie für das 1766 gefundene Titus-Bode-Gesetz ${ }^{9}$ oder Emmerich Zederbauers Die Harmonie im Weltall, in der Natur und Kunst (1917). ${ }^{10}$

Neben der Erforschung dieser auf naturwissenschaftliche Episteme gestützten Kosmographie begibt sich die Literatur und Philosophie des 18. Jahrhunderts auf die Suche nach einer Kosmographie des bewohnten Weltalls. Was liegt jenen Strukturen des bewohnten Kosmos zugrunde und aus welchen Konstituenten (jene strukturbedingenden Denkschablonen) werden sie konstruiert? Erstmals sind es astronomisch-physikalische Veranlagungen eines Himmelskörpers, die als äussere Konstituenten nicht nur metonymisch auf biologische und geistige Beschaffenheit der Planetenbewohner hinweisen, sondern sie auch ontisch präorganisieren."

Als Folge des Newtonschen Gravitationsgesetzes sieht Immanuel Kant in seiner Allgemeinen Naturgeschichte und Philosophie des Himmels einen direkten Zusammenhang zwischen der Wirkung der Gravitationskraft — und somit der Entfernung vom Gravitationszentrum eines Sonnensystems - und 
어 dem Vollkommenheitsgrad der Planetenbewohner. ${ }^{12}$ Die Gravitation ordnet den kosmischen Raum, indem sie den feinen vom groben Stoff aussondert. ${ }^{13}$ Eine solche Aussonderung der Stoffe hat wiederum zur Folge, dass unterschiedliche Räume als potenziell bewohnbare Topoi in Bezug auf das geistige Vermögen ihrer denkbaren Bewohner kein unbeschränktes, sondern ein bereits ausdifferenziertes geistiges Potenzial aufweisen. Denn je feiner und leichter ein Stoff ist, desto entfernter ist er vom Gravitationszentrum (der Sonne) verortet. Von der Feinheit des Stoffes, aus dem ein Lebewesen gebaut ist, hängt wiederum die Vollkommenheit des Geistes ab, der einen stofflichen Körper bewohnt. Die naturwissenschaftlich gestützte Kartographierung der Materie im Kosmos mündet somit in einer Fragmentierung und Strukturierung des Raumes hinsichtlich seines Schöpfungspotenzials. Auf einem Planeten kann sich nicht jede denkbare, sondern nur eine durch den Grad seiner materiellen Feinheit prädestinierte Form der geistigen Perfektion ausbilden. Es entsteht somit eine Hierarchie der kosmischen Räume, deren einzelne Stufen (das sind die ein$x$ zelnen Planeten) mit einem differierenden Potenzial der geistigen Veranlagung ihrer Lebewesen präorganisiert werden.

Insofern steht die literarische, durch die Rhetorik der Entmaterialisierung geprägte Darstellung der extraterrestrischen Lebewesen - etwa in Joseph Nürnbergers Roman Astronomische Reiseberichte oder Topographie des Himmels und planetarischen Metempsychose (1837) ${ }^{14}$ oder in dem anonym erschienenen Roman Zamor oder der Mann aus dem Monde - kein blosser Roman (1787) ${ }^{15}$ - in einem bipolaren Verhältnis zwischen der platonischen Denktradition der Entmaterialisierung ${ }^{16}$ und der Extrapolation der physikalischen Gesetze auf die Konstituenten der Weltenpluralität. Denn auch wenn die epistemisch-wissenschaftliche Grundlage für jene Fragmentierung der potenziell omnipotenten kosmischen Biosphäre wohl erst durch Kants und Laplaces Nebularhypothese entstanden ist, so lässt sich ihre philosophisch-kosmologische Prämisse dennoch in der Antike situieren. So hat der Geist-Materie-Dualismus seine Wurzeln in der platonischen und aristotelischen Kosmographie, gemäss welcher der Weltraum bekanntlich in Sphären unterschiedlicher Vollkommenheit gegliedert 


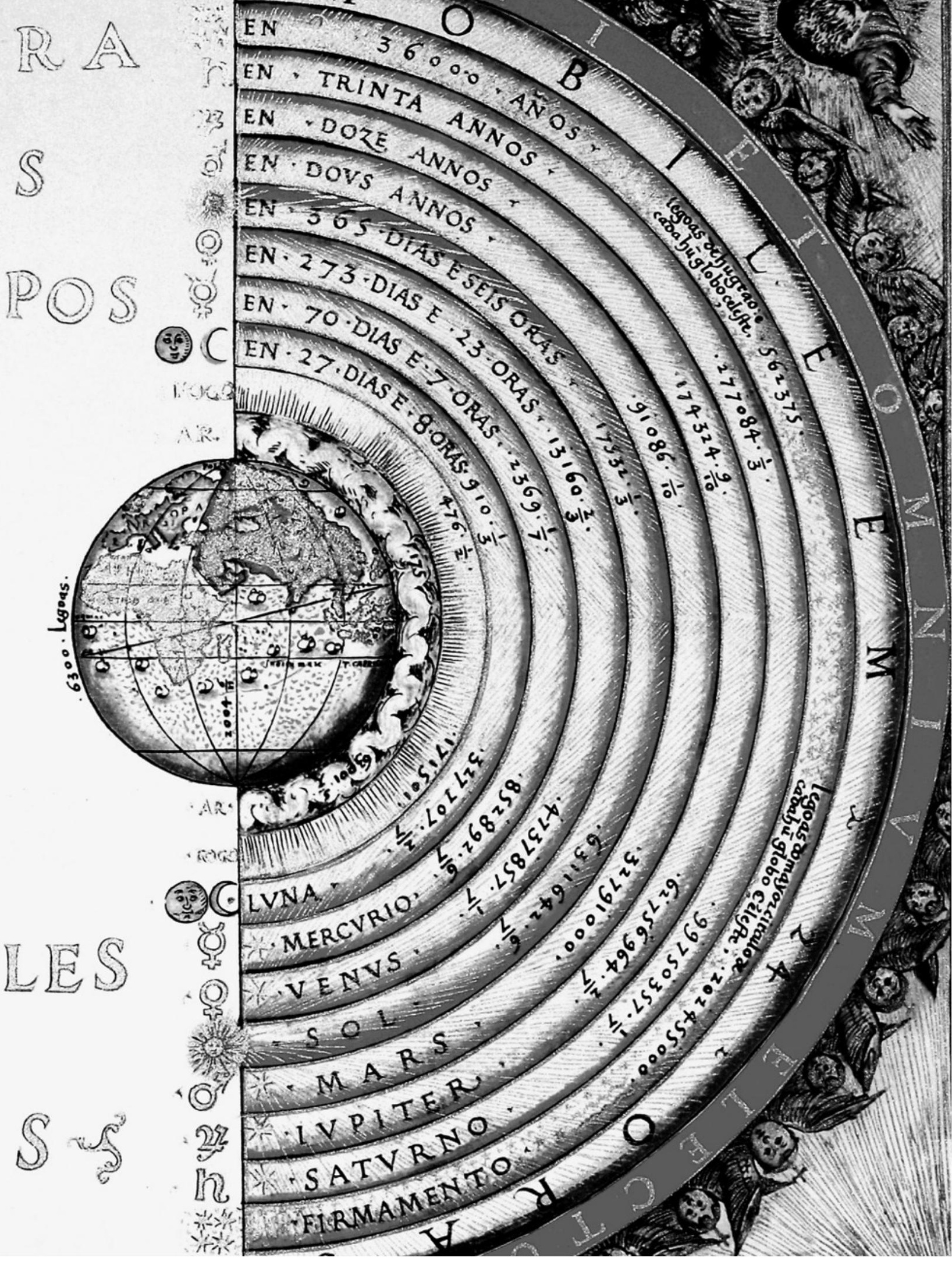


ㅇ wurde: ${ }^{17}$ maximal vollkommene Topoi der Vergeistigung (äussere Sphären) und minimal vollkommene Plätze der Materialisierung (Mittelpunkt der Erde). Auch hier sind die Erde und die einzelnen Himmelskörper hinsichtlich ihres ontischen Vermögens nicht homogen beschaffen, sondern je nach materieller - oder eben entmaterialisierter - Veranlagung kartographiert. Gemeinsam bilden sie ein System, eine Topographie von absteigender Materialität und aufsteigender Geistigkeit. Jede Sphäre erhält durch diese Kartographierung einen eigenen Wert in der Skala der Raumvergeistigung. Und obwohl die Planeten aufgrund ihrer ätherischen Beschaffenheit einen anderen ontischen Status als die Erde besitzen, sind sie schon bei Platon als Biosphären (bewohnte Räume) verstanden, die von den Seelen auf dem Wege ihrer geistigen Vervollkommnung durchwandert werden. ${ }^{18}$ Der Aufstieg der Seele vollzieht sich durch den Aufstieg in der Hierarchie der kosmischen Räume. Im geozentrischen Weltbild der aristotelischen Kosmologie, die die mittelalterliche Denktradition richtungsweisend beeinflusst hat, wird das «Mass der Entfernung der Sphären [...] zum Kri-

terium der Wertigkeit».. ${ }^{9}$ Der Raum wird nicht nur nach seiner geistigen und materiellen Beschaffenheit seziert, sondern auch moralisiert. Zu den wohl bekanntesten literarischen Erörterungen jener kosmologischen Vorstellungen der präkopernikanischen Ära gehört Dantes Divina commedia (1321), eine Ichwanderung von den untersten Stufen der Vergeistigung (Mond) bis hin zu den Orten der höheren Seelengradation (den Sphären der äusseren Planeten). ${ }^{20}$

Mit dem Untergang des Geozentrismus - so die Ausgangsthese unserer Beobachtung - entsteht zwar eine neue räumliche Struktur des physikalischen Kosmos, die Konstituenten, die die Ordnung des bewohnten Weltraums bestimmen, gleichen allerdings den Prinzipien, nach denen die aristotelische und platonische Kosmographie kartographiert wurde. ${ }^{21}$ Es handelt sich um die im Zusammenhang mit antiken Kosmologien erwähnten Konstituenten I. der moralischen Wertigkeit, II. der materiellen Beschaffenheit und III. der paradigmatischen Funktionsweise des Weltalls. Beispielsweise beschreibt Johann Gottfried Herder in seiner Abhandlung Über die Seelenwanderung (1782) «unser Sternengebäude» als ein 
ㄱ «vom letzten Planeten bis zur Sonne hinauf» aufsteigendes System der «Gradation» von Schöpfung, Licht und physikalischen Kräften:

[...] setzen Sie die Sonne nun als den grossen Versammlungsort aller Wesen des Systems, das sie beherrscht, so wie sie ja auch die Königin des Lichts und aller Wärme, aller Schönheit und Wahrheit ist, die überall den Geschöpfen gradweise mitteilt. [...] Je entfernter von unserer Sonne, desto dunkler, desto gröber; je näher desto heller, leichter, wärmer, geschwinder. Die Geschöpfe des Merkur, der immer in den Strahlen der Sonne verborgen ist, müssen freilich von andrer Art sein, als jene trägen Saturnus-Bewohner $[\ldots]^{22}$

Die Sonne ist dabei der leichteste, hellste und vollkommenste Ort, und der Grad dieser Beschaffenheiten steigt proportional zur räumlichen Entfernung vom Tagesgestirn ab. Jene vollen-

deten Räume sind wiederum von Lebewesen differierender Gradation der Aurea Catena Homeri bewohnt. In Thomas Wrights An Original Theory or New Hypothesis of the Universe $(1734)^{23}$ ist hingegen nicht unsere Sonne der Ort des absoluten Ens perfectissimum, sondern der thronende Gott höchstpersönlich, um den die unzähligen Welten kreisen, deren Perfektionsgrad mit der räumlichen Entfernung vom absoluten Zentrum abnimmt. Auch Wielands Lehrgedicht Die Natur der Dinge in sechs Büchern (1752) stellt ein Universum vor, in dem jeder Planet als Wohnort einer anderen Stufe der Vergeistigung präorganisiert wurde..$^{24}$ Gottesnahe Himmelskörper «bei den Sternen» sind - ganz im Sinne der antiken Kosmologien eines Aristoteles oder Platons - Wohnplätze der maximal vergeistigten Lebewesen, während gottesferne Räume metonymisch auf die materielle Grobheit und moralische Unreife ${ }^{25}$ der dortigen Bewohner verweisen. ${ }^{26}$ Wie der «Dunst» aufsteigt und sich vom «niedrigen Morast» befreit, so befreien sich ununterbrochen die Seelen von der Last der Materie und entwickeln sich moralisch, indem sie den kosmischen Raum durchwandern, sich von den Räumen der materiellen Grobheit entfernen und 
$\bar{N}$ immer mehr vergeistigte und gottesnähere Orte bewohnen. ${ }^{27}$ In Johann Christoph Röhlings Roman Reise eines Marsbewohners auf die Erde (1791) träumt der Protagonist von den "höhere[n] Region[en]» des Kosmos. Auch hier bildet das Universum ein System der planetarischen Palingenesie, der Wiedergeburt der Seelen auf fremden, ihrem Läuterungsrang zugeordneten Planeten.$^{28}$ Wie bei Aristoteles oder Platon sind somit auch Herders, Wrights, Wielands oder Röhlings Kosmologien mit ethischen (Wertigkeit) ${ }^{29}$ und stofflichen (Entmaterialisierung) Konstituenten umschrieben. Und auch hier sind die Himmelskörper in Bezug auf den Grad der Wertigkeit und Entmaterialisierung nicht homogen organisiert. Vielmehr wird ihnen durch die schon in Platons Timaios (ca.360 v. Chr.) diskutierte, teleologische Notwendigkeit des Vorhandenseins aller möglichen Graduierungen im Universum ${ }^{30}$ nur ein jeweils bestimmter und bei jedem Planeten unterschiedlicher Grad der stofflichen Beschaffenheit und ethischen Präorganisation zugeschrieben. Der Kosmos ist ein Paradigma, ein korrelierendes System, dessen Bestandteile (diverse Himmelskörper)

jeweils andere Funktionen als Wohnorte bestimmter Gradationen der Lebewesen übernehmen.

\section{$\odot \odot$ \\ ÄSTHETIK \\ EXTRATERRESTRISCHER RÄUME}

Diese Interdependenz zwischen den die antiken Kosmographien beschreibenden Konstituenten und dem bewohnbaren, postkopernikanischen Universum drückt sich auch in der literarischen Darstellungsweise der extraterrestrischen Räume aus. Zu zeigen ist, dass die Schilderungen fremder Planeten in der Literatur um 1800 auf die alten, mittelalterlichen Beschreibungsmuster des Himmels bzw. der Hölle zurückgreifen, die wiederum an die erwähnten Konstituenten der antiken Kosmologien (moralische Wertigkeit, Entmaterialisierung usw.) anschliessen.

A) Entmaterialisierung vs. Finsternis

«Mein Geist hat seine körperliche Hülle verlassen und durcheilt den Weltraum» - so beginnt die junge Somnambule 
N Iphigenia ihren extraterrestrischen Reisebericht in Dr. Rudolphios Buch Die junge Hellseherin. Neue Mittheilungen der Somnambule Iphigenia Strudella über das Jenseits, von ihren Reisen in den Mond, die Sonne und die Sterne (1867). ${ }^{31}$ Das All erweist sich dabei - wie in vielen ähnlichen extraterrestrischen Reiseberichten dieser Zeit, etwa dem schon erwähnten Zamor oder der Mann aus dem Monde - kein blosser Roman (1787), Philippine Demuth-Bäurles 1834 erschienenen Reisen in den Mond, in mehrere Sterne und in die Sonne ${ }^{32}$ oder dem von Friedrich Ebner verfassten Roman Neue Reisen in den Mond, die Planeten, Sonne und andere Sterne ${ }^{33}$ - als Raum der christlichen Läuterung. Jeder Planet beherbergt eine andere Gradationsstufe der Lebewesen, die sich auf dem Wege der planetarischen Palingenesie dem Ort der höchsten Seligkeit, der göttlichen Sonne, räumlich immer weiter annähern. ${ }^{34}$ Die moralische Erziehung des Menschen bedeutet die Wiedergeburt auf einem neuen, dem Ort der maximalen göttlichen Perfektion näheren Planeten. Auch hier ist der Einfluss der antiken Kosmologie kaum zu übersehen, der vor allem dann deutlich wird, wenn

der moralische und räumliche Aufstieg der Seelen im Prozess der Palingenesie durch die zunehmende Entmaterialisierung bzw. Vergeistigung der Räume und ihrer Bewohner begleitet wird. Die Räume der Unseligen sind zunehmend materialisiert (Graduierung des Höllischen), während die Himmelskörper der Vollkommenen immer mehr als vergeistigt dargestellt werden (Graduierung des Himmlischen). Diese uralte kosmologische Prämisse prägt schon die Darstellung des Himmels und der Hölle in der mittelalterlichen heilsgeschichtlichen Literaturtradition - etwa im Text Himmel und Hölle (anonymer Verfasser, um 1090) ${ }^{35}$ Sie bildet auch die Grundlage für die Schilderung der Destinationsorte der reinen und der verfallenen Seelen in der extraterrestrischen Literatur des 18. und frühen 19. Jahrhunderts. Im Folgenden sollen aus den erwähnten Quellen Textstellen zitiert werden, welche die zeittypische Darstellungsweise exoplanetarischer Landschaften exemplifizieren:

Die Planeten der Seligen:

«Er hat mit unserer Erde viel Aehnlichkeit, nur ist er viel lichter, feiner und milderı $;{ }^{36}$ 
«Sein Angesicht ist hellglänzenderals die Sonne, sein Auge feurig und doch voll Liebe [...]»;37

«Es ist aus Steinen gebaut, aber auf unserer Welt habe ich noch keine der Art gesehen, sie sind durchscheinend hell weiss. ${ }^{38}{ }^{38}$

«Alles ist ganz anders, lichter, feiner, erhabener, vollkommenerı; ${ }^{39}$

«Ist Pallas noch schöner als Mars? Antwort: Gewiss, unvergleichlich schöner [...]. Ihre Schönheit und das Strahlende ihrer Gewänder über die der Marsbewohner ist ausserordentlich; nicht ein Mal die Lehrer auf dem Mars sind so schön ; $^{40}$

«[...] ich bin in einer Stadt, so prächtig, dass alle Zungen der Menschen nicht hinreichen würden, sie zu schildern. Alles strahlt von himmlischem, ja ich möchte sagen göttlichem Glanze»; ${ }^{41}$

«Die Kleider gleichen immer denjenigen, welche ich früher schon beschrieben habe, allein je höher der Grad, um so schöner werden sie, sie strahlen weit glänzender und erhabener, als nach dem irdischen Auge die Sonne»; ${ }^{42}$

"Wie ist das Aussehen der Saturnsbewohner? Antwort: Sie sind so glänzend und klar, dass sie fast durchsichtig sind $» ; ;^{43}$

«Ja. Die Städte haben ja vierzehn Thore; diese sind, wie die Mauern, Häuser und alle anderen Gegenstände, halb durchsichtig und ausserordentlich klar. Die Pflanzen tragen eine erstaunliche Farbenpracht zur Schau, es ist mir aber, als wenn sie nur von Luft oder von einer noch viel feinern Materie seien, denn die meisten sind durchsichtig, alle aber halb durchsichtig. - Die Bewohner des Uranus tragen wallende Gewänder, sie sind jedoch so zart, so ätherisch, dass ich immer glaube, sie müssten sich vor meinen Augen in Nichts auflösen. $" ;^{44}$

«Frage: Ist der Ort, wo du jetzt weilst und den du Mond nennst, sehr verschieden von der Erde? Antwort: er ist ungleich schöner und grossartiger, jeder Gegenstand ist fast durchsichtig und licht, der Boden 


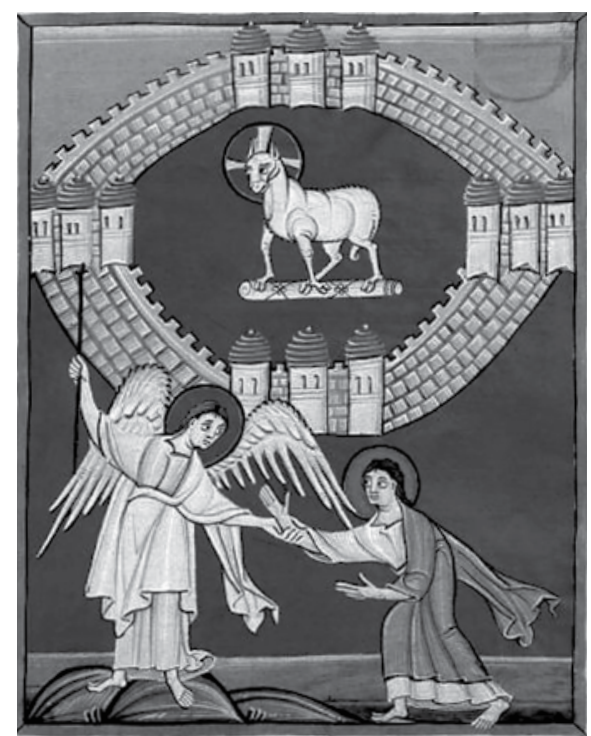

A B B. I I

«DAS NEUE JERUSALEM». MINIATUR NR. 49 AUS DER BAMBERGER APOKALYPSE (UM 1000). MINIATUR AUF GOLDGRUND. 
Die Planeten der gefallenen Seelen:

"die Finsterniss so gross und so dick sey, dass solche mit den Händen ergriffen werden könne» ${ }^{46}$

"Diesen Ort bezeichnete sie wieder als ein Thal, grösser, finsterer und kälter als das vorige ; $^{47}$

«Frage: Von welchem Umfang und welcher Beschaffenheit scheinen dir diese unbekannten Welten zu sein? Antwort: Sehr verschieden. Während einige durchsichtig und glänzend erscheinen, sind andere in Nebeloder Wolken gehüllt» [Hervorhebungen: MC]. ${ }^{48}$

Das Prinzip der Entmaterialisierung strukturierte schon den kosmischen Raum der antiken Kosmologien, die nach Richard Goerwitz zu Beginn des 11. Jahrhunderts mit der biblischen Exegese verschmelzen ${ }^{49}$ und zur Grundlage der kirchlichen Weltalllehre für die nächsten Jahrhunderte werden. Bevor allerdings jene Gliederung des antiken Kosmos in ein Spektrum zweier entgegengesetzter Räume, eines Topos der Vergeistigung und eines der materiellen Grobheit, zur Ordnungskonstituente des bewohnten Weltalls in der postkopernikanischen Literatur avanciert, prägt sie fundamental schon in der frühmittelhochdeutschen Texttradition die Darstellung der Hölle und des himmlischen Jerusalems. Wie die Planeten der gefallenen Seelen in Dr. Rudolphios oder Demuth-Bäurles extraterrestrischen Reiseberichten sich als Orte der «in Nebel oder Wolken gehüllt[en]» «Finsterniss» erweisen, so ist auch im mittelalterlichen Lehrgedicht Himmel und Hölle die Unterwelt als finsteres Nebelreich dargestellt («verwazzenlich genibile[...] egilich vinster»). ${ }^{50}$ Die Seligen auf den für sie prädestinierten Planeten ${ }^{51}$ sowie ihre Städte und Kleider sind hingegen, wie aus den obigen Zitaten zu entnehmen ist, durchsichtig, glänzend und «ausserordentlich klar», genauso wie das himmlische Jerusalem «durhscôuvig» und "durhluther» erscheint. ${ }^{52}$ Diese narrative Nähe der beiden Texttraditionen in Bezug auf eine Ästhetik der Entmaterialisierung zeigt sich besonders deutlich an der Negation der materiellen Kostbarkeit der plane- 
$\stackrel{N}{ }$ tarischen respektive himmlischen Architektur, die zwar aus gold- oder diamantenähnlichen Mineralien gemacht ist, jedoch in ihrem Wesen durchsichtig und hellglänzend erscheint. Die architektonische Landschaft ist sowohl im mittelalterlichen himmlischen Jerusalem wie auch in den extraterrestrischen Städten aus Materialien gebaut, die «wie Gold» glänzen und «wie Diamanten» «klar» sind. ${ }^{53}$ Die Äquivalenz zwischen den materiellen Kostbarkeiten (Gold, Diamanten) und ihrer himmlischen bzw. extraterrestrischen Entsprechung («wie Gold», «wie Diamanten») beschränkt sich ausschliesslich auf das Tertium Comparationis der hohen Wertigkeit per se. Die materielle Komponente jener Wertigkeit wird hingegen in Darstellungen des Himmels und der vollkommenen Planeten durch die Rhetorik der Entmaterialisierung konsequent negiert («erbaut von durchsichtigen Stoffen»). ${ }^{54}$

Die Opposition zwischen den vergeistigten Sphären des Vollendeten und den materialisierten Räumen des Unvollkommenen prägt die kosmographische Verortung und Darstellungsweise des Himmels und der Hölle. Sie avanciert dann in der postkopernikanischen Ära zur kosmologischen, das bewohnte Weltall strukturierenden Konstituente (etwa bei Herder, Wright, Wieland), und gestaltet somit eine dem Himmlischen respektive Höllischen analoge, auf die Rhetorik der Ent- bzw. Materialisierung gestützte Darstellungsweise der bewohnten Planeten.

\section{B) Ordnung, Gleichförmigkeit vs. Kontingenz}

Nicht nur die Rhetorik der Entmaterialisierung, sondern auch die mathematisch beschreibbare Ordnung der Architektur- oder Naturlandschaft gehört zu den konventionalisierten Darstellungsmustern des himmlischen Jerusalems. ${ }^{55}$ Auch hier ist der Ursprung jener Ästhetik des Geometrischen in den kosmologischen Vorbildern eines Pythagoras und eines Platons zu finden, der die Schöpfung des Demiurgs als eine durchdachte, mathematische Struktur verstand (vgl. etwa die Proportionen der Sphären in Timaios). Seit dieser Zeit sind die Ästhetik und Geometrie kaum voneinander zu trennen, und der Gipfelpunkt dieser Symbiose manifestiert sich in der Poiesis des Allerschönsten und Geordneten, nämlich in der 
ล Darstellung des himmlischen Jerusalems. Mit der Kosmopoetik wird hier nicht etwa eine Poetik des Kosmischen, sondern die Konditionalisierung des Poetischen durch die ästhetisierte Kosmologie gemeint. Um es einfach auszudrücken: Es handelt sich hier um die Geburt der Kunst aus dem Geiste des Weltalls, da der Künstler nach denselben Poiesis-Kräften der geometrischen Ästhetik handelt, mittels derer Gott die souveräne Schöpfung seines allergrössten Kunstwerkes, des Weltalls, vollbracht hat - eine Ansicht, die an prominentester Stelle in Keplers Harmonices Mundi vertreten wird. ${ }^{56}$

Jene kosmopoetisch inspirierte Symmetriesierung der Architektur, die Peter Jezler in seinem Buch Himmel, Hölle, Fegefeuer. Das Jenseits im Mittelalter als konventionalisiertes Darstellungsmuster des himmlischen Jerusalems in der mittelalterlichen Texttradition beschreibt, findet ebenfalls Widerhall in der Schilderungsweise der Planeten höherer Gradationsstufen:

Die Planeten der Seligen:

«Ich bin jetzt nicht nur im Mond, sondern mein Führer hat mich bereits in eine Stadt geführt; auf unserer Erde kommt dieser bei weitem keine an Schönheit gleich, die Gebäude sind gleich gross und schön $[\ldots] \| ;^{57}$

«Unten am Fusse des Berges, ist ein herrliches Wäldchen angelegt, die Bäume stehen alle in der schönsten Ordnung da [...]»; $;^{58}$

«[...]; die Bäume stehen in geordneten Reihen da, alle haben eine gleiche Höhe und Dicke [...]»; $;^{59}$

«[...] nämlich; dass die Gebäude in allen den Städten gleich sind, nur mit dem Unterschiede, dass sie immer schöner werden, je höher der Grad der Seligkeit steigt $» ;^{60}$

"[...] ob ich es denn noch nicht bemerkt habe, dass die Länge und Breite der Städte immergleich sey, alle sind viereckigt $\gg .{ }^{61}$

Gleiche Länge und Breite der Städte, einförmige architektonische Landschaft und sogar die Symmetrie der sonst kontin- 


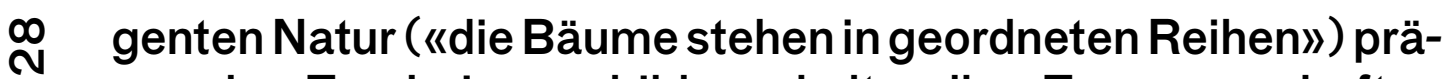
gen das Erscheinungsbild von kulturellen Errungenschaften der in der Aurea Catena Homeri höher situierten Planetenbewohner.

\section{C) Ästhetik des Akustischen}

Auch was die Musik anbelangt, zeigen sich weitreichende Interferenzen zwischen der mittelalterlichen Darstellungsweise des himmlischen Jerusalems und den Schilderungen des Kosmos um 1800. Die folgenden Zitate exemplifizieren die um 1800 gebräuchliche Beschreibung musikalischer Praktiken auf unterschiedlich graduierten Himmelskörpern, den Planeten des Ens perfectissimum und den extraterrestrischen Topoi der gefallenen Seelen:

\section{Die Planeten der Seligen:}

"[...] sagte ich nicht schon öfters, dass das Geschäfte der Seeligen nichts anders sey, als Singen, Beten, Gott, seinen Sohn und den heil. Geist verehren und lernen, dieses hört in ewige Ewigkeit nicht auf. Wirklich singen sie das Lied: 〈Herr Zebaoth, dich loben wir [*unlesbar].) Die Lehrer stehen oben an und sind die Vorsänger Wenn ich nur hier bleiben dürfte! aber das wird mir vor jezt verweigert. Wie herrlich der Gesang und die Musik ist, vermag ich gar nicht zu sagen $[\ldots] »{ }^{62}$

\section{Die Planeten der Unseligen:}

«Als sie nun in dem dritten Grad anlangte, da brach sie in einen Strom von Thränen und mehr als lautes Schluchzen und Seufzen aus [...]»; ${ }^{63}$

"Oede, felsig, verlassen und trostlos. Kein freundliches Plätzchen, kein liebliches Thal, kein schattiger Baum weit und breit. Nichts bietet Schutz gegen die glühende Hitze $[\ldots] »{ }^{64}$

Während die Seligen der fernen Welten wie die erlösten Engel im Himmel immer prachtvoller singend Gott preisen, erweisen sich die Orte der moralisch gefallenen und in der Hierarchie der 


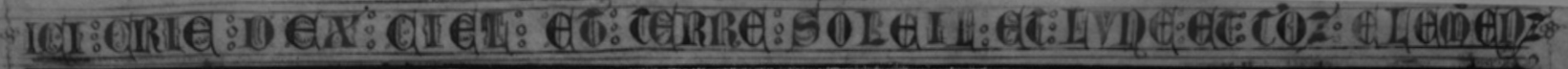

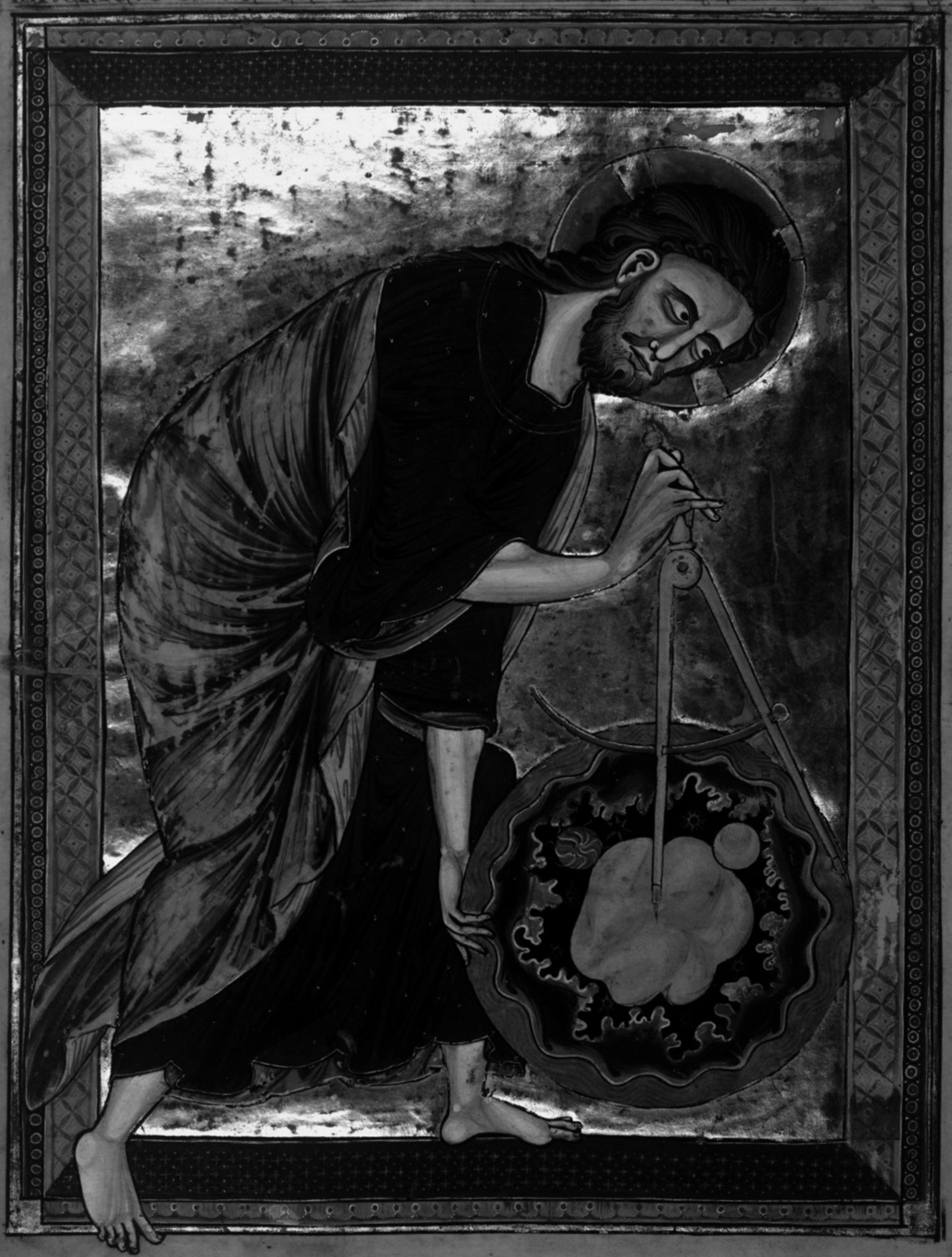


으 Schöpfung absteigenden Geister als Topoi des «Schluchzens und Seufzens». Die Analogie zur mittelalterlichen Hölle, welche die schöne Musik des Himmels antithetisch verneint und als Ort der «Wehklage und des Jammers» ("karôt unde jâmer») ${ }^{65}$ gilt, ist auch hier kaum zu übersehen. Doch schon lange vor der neuplatonischen Gradation des Himmlischen und Höllischen und ihrer Verortung im physikalischen (zu- oder abnehmend materialisierten) Weltall wird der Kosmos in theologischen Schriften etwa eines Eusebius Kaisareia oder Ambrosius zu musikalischen Instrument stilisiert. Durch die Sphärenmusik soll nun Gott, der Schöpfer, in seiner Allmacht und Allherrlichkeit gepriesen werden. ${ }^{66}$ In der neuplatonischen Kosmologie um 1800 verliert das Weltall zwar seine musikalische Natur, es wird aber wie das himmlische Jerusalem zum Ort des paradiesischen Musizierens und zugleich zum Topos der höllischen Klagen. Kein musikalisches Instrument soll das Weltall sein, sondern die Kulisse und Bühne, auf der das Konzert der neugeborenen Seelen auf dem Wege zur Vollkommenheit stattfindet.

\section{$\odot \odot \odot$ \\ POETIK DER NEGIERTEN FIKTIONALITÄT}

Die obige Analyse zeigt unterschiedliche Interdependenzen zwischen der mittelalterlichen Himmels- und Höllendarstellung sowie der Imagination der extraterrestrischen Räume in der Literatur um 1800. Dabei erweist sich die Äquivalenz der konventionalisierten Darstellungsweisen des mittelalterlichen Jenseits und der ausserirdischen Topoi als eine durch die antike Kosmologie und Ästhetik (Rhetorik der Entmaterialisierung und Ästhetik der Symmetrisierung) geprägte narrative Konstruktion. Jene Divergenz zwischen der naturwissenschaftlichen Implementierung des Kosmos, die seit Kopernikus neue Raumstrukturen hervorbringt, und der Kosmologie des imaginierten bewohnten Weltalls, deren Kosmographien auf platonischaristotelischen Konstituenten der zunehmenden Entmaterialisierung und Ästhetisierung basieren $(\mathrm{vgl} . \odot)$, spiegelt sich auch in der Poetik der zeitgenössischen Weltraumliteratur wider.

Einerseits ist nämlich die fiktionale Hierarchisierung der bewohnten Planeten durch die erwähnten kosmologischen Konstituenten geprägt. Jedem Himmelskörper wird ein Wert 
$\bar{m}$ in der Hierarchie der bewohnten Räume zugeordnet, abhängig davon, mit welchen Lebewesen jener Planet bewohnt ist. Somit entsteht eine Hierarchie bzw. Kosmographie des bewohnten Weltalls, die nicht primär durch physikalische Gesetze determiniert wird, sondern durch Prämissen, die die Hierarchie der Lebewesen präorganisieren: eine zunehmende Moralisierung und Vergeistigung. So entsteht in vielen zeitgenössischen Texten eine Kosmographie des bewohnten Weltraums, die durch unterschiedliche Gradationen der Planeten beschrieben werden kann, je nachdem, welchen Platz seine Bewohner in der Aurea Catena Homeri einnehmen. Je höher solche Lebewesen in der Hierarchie eingestuft werden, desto moralischer und geistiger sind sie. Moralisierung und Vergeistigung konstituieren eine Hierarchie der Lebewesen, die dann wiederum in den Weltraum projiziert wird und eine Kosmographie des bewohnten Weltalls hervorbringt. Jene Projektion der Aurea Catena Homeri setzt allerdings voraus, dass die einzelnen Planeten als Wohnorte eben nicht omnipotent sind, sondern nur eine bestimmte Gradationsstufe der Lebewesen beherbergen kön-

nen. Jeder Ort entspricht einem Grad der Lebewesen und beansprucht für sich den seinen Bewohnern zugeteilten Rang der Entmaterialisierung und Moralisierung. Die Etablierung einer solchen Hierarchie der Lebewesen ist wiederum als Produkt der antiken Kosmologien zu betrachten, die den Weltraum als ein Spektrum zweier entgegengesetzter Räume begreifen, die in Bezug auf ihre materielle Beschaffenheit und moralische Prädisposition miteinander konkurrieren. Dieselben Konstituenten, die das mittelalterliche Weltbild stützen, determinieren somit auch die Struktur des bewohnten Kosmos, wie sie die Prosa des 18. Jahrhunderts imaginiert - wofür nicht zuletzt auch die oft dargestellte Analogie von extraterrestrischen Räumen und himmlischem Jerusalem spricht.

Andererseits interferieren jene traditionellen Raumbeschreibungsmodelle mit zeitgenössischen Wissensdiskursen der postkopernikanischen Weltanschauung. So ist zwar das Bild des bewohnten Kosmos in Friedrich Ebners Roman Neue Reisen in den Mond, die Planeten, Sonne und andere Sterne durch mittelalterliche Darstellungsmuster der himmlischen Paradiese weitreichend determiniert und somit auf die Kosmo- 
กิ logie der Entmaterialisierung und Moralisierung gestützt, die Darstellung der Natur des physikalischen Kosmos orientiert sich aber am zeitgenössischen Wissensdiskurs. Jeder Besuch der Somnambule Marie auf einem Planeten beginnt mit der Präsentation des astronomischen Wissens über jenen Himmelskörper, ${ }^{67}$ die als empirische Bestätigung der durch die irdischen Astronomen aufgestellten wissenschaftlichen Spekulationen inszeniert wird. Die Marie reist durch das Universum, (auch) um unsere epistemischen Annahmen und Hypothesen empirisch zu überprüfen. Deutlich wird dieser Anspruch des Textes, wenn die physikalischen Angaben zu unserem irdischen Trabanten, den Marie zusammen mit ihrem Führer besucht, mit Fussnoten versehen werden, die die epistemischen Forderungen des Textes untermauern sollen: «Diese Angaben stimmen mit den von den Gelehrten aufgestellten Berechnungen vollkommen überein; wo dies nicht der Fall sein sollte, werden wir die Angaben der Astronomen in Anmerkungen aufführen. ${ }^{68}$ Erst nach dieser Selbstinszenierung des Textes als wissenschaftlich-empirischer Bericht folgen die Beschreibungen der immer schöneren Städte und der immer vergeistigteren Bewohner, die wiederum auf die traditionellen Beschreibungsmodelle der Kosmologie rekurrieren.

Die Einbettung der Narration in den postkopernikanischen Wissenschaftsdiskurs wertet den epistemischen Modus der Erzählung auf. Dazu trägt allerdings nicht nur die Selbstinszenierung des Textes als wissenschaftlich-empirische Expedition bei, sondern auch (oder vor allem) die Einbettung der Erzählung in einen selbstkonstruierten, medialen Rahmen. So ist Dr. Rudolphios Roman Die junge Hellseherin nicht etwa als Produkt der Imaginationskraft zu lesen, sondern als «Mittheilungen» Iphigenia Strudellas über ihre somnambule Reise durch das Universum: ${ }^{69}$

\footnotetext{
Die Vorsehung hat uns nicht allein in der heiligen Schrift darüber einigen Aufschluss gegeben, woran wir glauben müssen, wenn wir wollen seelig werden, sondern sie hat hin und wieder Menschen mit einer Sehergabe beschenkt, welche Blicke in die räthselhafte Zukunft des Jenseits zu werfen gestattete. Zu
} 
den merkwürdigen Erscheinungen dieser Art gehört unstreitig der Somnambulismus, bei dem sich der Geist gleichsam vom Körper trennt, in höhere Regionen entschwebt und die dabei gemachten Beobachtungen gewissen Personen mittheilt, welche dazu eine eigene Disposition nöthig zu haben scheinen. ${ }^{70}$

In der danach folgenden Einleitung wird «das Wesen des wahren Somnambulismus» erläutert, der als Wirkung des tierischen Magnetismus zwar wissenschaftlich nicht ganz unumstritten war, aber dennoch einen fixen Platz in den damaligen szientifischen Diskussionen fand. War einst der Traum ein Signal für den fiktionalen Modus der Erzählung - so etwa in Keplers Somnium (1634) -, so avanciert er durch die pseudowissenschaftlichen Erkenntnisse des Mesmerismus zur epistemischen Beglaubigungsstrategie des Erzählten.

Cum anno 1608 ferverent dissidia inter fratres Imperatorem Rudolphum et Matthiam Archiducem, eorumque actiones vulgo ad exempla referrent ex historia Bohemica petita, ego publica vulgi curiositate excitus ad Bohemica legenda animum appuli. Cumque incidissem in historiam Libussae virginis, arte magica celebratissimae, factum (est) quadam nocte, ut post contemplationem siderum et Lunae lecto compositus altius obdormiscerem, atque mihi per somnum visus sum librum ex nundinis allatum perlegere, cuius hic erat tenor. ${ }^{71}$

Bereits in der Einleitung des Somnium führt Kepler eine fundamentale Koinzidenz herbei: Es entsteht der Eindruck, als ob der Erzähler den epistemischen Status der Narration durch eine geradezu überbetonte fiktionale Mediatisierung des Erzählten um jeden Preis negieren wolle, indem er das Beschriebene als ein Wissen der in der Allgemeinheit für Erzeugungsmittel der Fiktionalität geltenden Medien inszeniert (etwa Buch oder Traum). Schon in diesen ersten Sätzen stellt sich heraus, dass die Erzählung eine Nacherzählung dessen ist, was der Narrator in einem Buch gelesen hat. Mehr noch: Jene literarische 


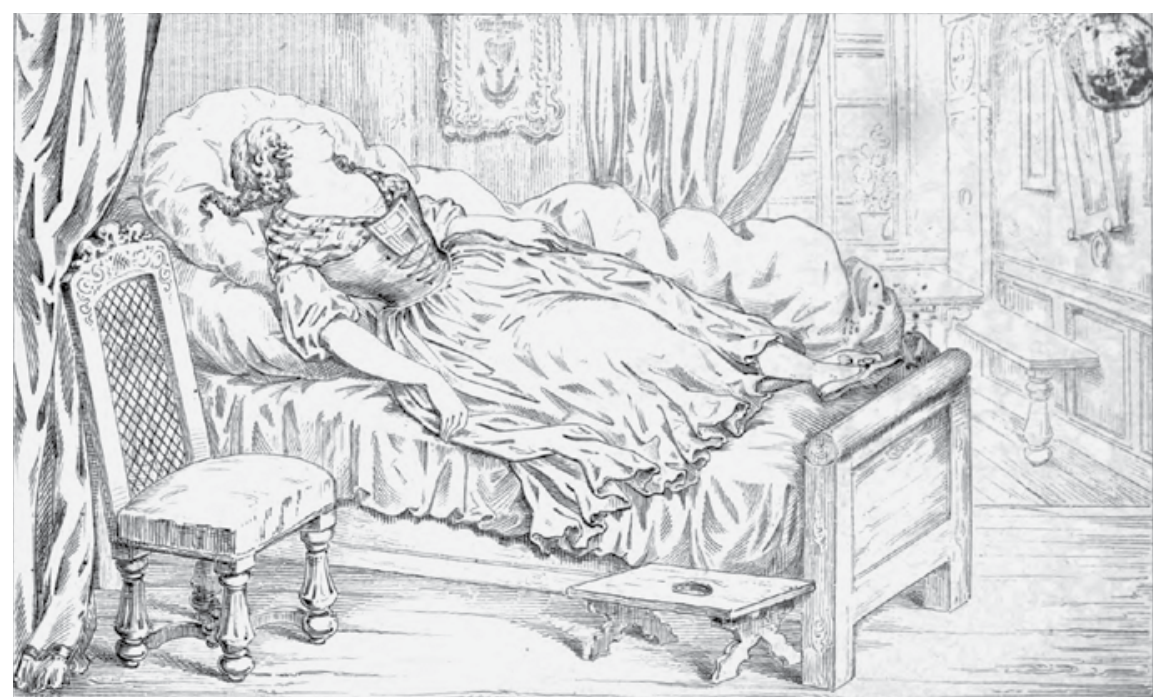

A B B. I V

EINE SCHLAFENDE SOMNAMBULE. FRONTISPIZ ZU FRIEDRICH EBNERS NEUE REISEN IN DEN MOND, DIE PLANETEN, SONNE UND ANDERE STERNE: AUS DEM LEBEN DER SOMNAMBÜLE MARIE W....... VON U........, AUF DEM SCHWARZWALDE: MERKWÜRDIGE ENTHÜLLUNGEN AUS DEM GEISTERREICHE; NEBST EINEM ANHANGE ERPROPTER HEILMITTEL UND MAGNETO-ELECTRICITÄT ZUR HEILUNG ALLER NERVÖSEN KRANKHEITEN (1852). 
L 2 Lektüre ist nur ein geträumter Anschein, der nie hätte entstehen können, wäre sein Geist in jener Nacht durch die Geschichte der Zauberin Libussa und durch die Betrachtung der extraterrestrischen Räume nicht «für Höheres empfänglich» gewesen. Kurz und bündig: Ein empfindlicher Geist träumt von der Lektüre, die nun in Form der folgenden Erzählung wiedergegeben werden soll. Es stellt sich die Frage, welchem Zweck jene $\mathrm{Hy}$ permediatisierung, jene Inszenierung der Erzählung als Artefakt der Fiktionsmedien dienen soll. Vielleicht wird im Text durch solche medialen Multi-Interferenzen eine poetische Konstruktion entworfen, deren Ziel darin besteht, die Reise durch den kopernikanischen Kosmos als blosse Fiktion epistemisch abzuwerten und von den wissenschaftlich unumstrittenen Fakten im Buch klar abzutrennen. ${ }^{72}$

Ist der Traum bei Kepler als narrativer Mechanismus anzusehen, um den fiktionalen Modus der Erzählung als solchen zu unterstreichen, so wird seine Bedeutung für die literarische Kommunikation unter dem Einfluss des Mesmerismus und der romantischen Überzeugung vom epistemischen Wert des Träumens - etwa in Novalis' Heinrich von Ofterdingen $(1802)^{73}$ - neu positioniert. ${ }^{74}$ Der somnambule Traum erscheint als ein Mittel der höheren Erkenntnis, das nun in vielen Werken extraterrestrischer Fiktion als Instrument der literarischen Kommunikation eingesetzt wird, um die Narration epistemisch aufzuwerten und somit die Selbstinszenierung des Textes, seine poetische Maske, zu gestalten. So kann Friedrich Ebners Roman Neue Reisen in den Mond, die Planeten, Sonne und andere Sterne auf die populärwissenschaftliche Fundierung des Somnambulismus nicht verzichten, ohne den Maries Erzählung als extraterrestrischer Bericht nicht glaubwürdig wäre. ${ }^{75}$ Der Text antizipiert einen medialen Rahmen (somnambuler Traum), der durch eine populärwissenschaftliche Fundierung (Mesmerismus) als Medium der epistemischen Erkenntnis aufgewertet wird, und inszeniert sich selbst als ein Wissen, das durch jene vorweggenommene Mediatisierung bedingt wird (Bericht). Keplers Somnium und die somnambulen Romane zeigen einerseits die Relevanz des Traumes für die extraterrestrische Poetik, denn die literarische Kommunikation inszeniert sich in beiden Fällen als eine durch 


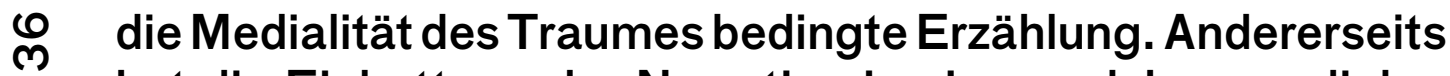
hat die Einbettung der Narration in einen solchen medialen Rahmen aber auch unterschiedliche Konsequenzen für die Selbstinszenierung des Textes. Denn während in Keplers Somnium der Traum als ein Glied der medialen Hyperbel fungiert, die den fiktionalen Charakter der Mondreise geradezu überbetonen soll, wird der Traum in den somnambulen Romanen als ein wissenschaftlich fundiertes Medium inszeniert. Somit entwirft der Text eine Maske für sich, die seinen fiktionalen Status verdecken soll. Was auf der Oberfläche erscheint, ist ein selbstinszeniertes Erscheinungsbild des Textes als extraterrestrischer Bericht.

Die Poetik der negierten Fiktionalität — so liesse sich dieses literarisch-kommunikative Verfahren bezeichnen - beruht auf der literarischen Konstruktion medialer Verschaltungen, die der vermittelten extraterrestrischen Welt einen epistemischen Status verleihen sollen. Diese Konstruktion kann, wie die zurückliegenden Beispiele gezeigt haben, unterschiedliche Gestalt annehmen: die Inszenierung der Erzählung als wissenschaftlich-empirischer Revisionsbericht oder als ein durch ein szientifisch fundiertes Medium vermitteltes Wissen. Hieraus resultiert noch eine weitere Beobachtung: Um die poetische Phantasterei zu verdecken und sich als Erkenntnis zu inszenieren, braucht die Weltraumliteratur einen glaubwürdigen medialen oder kommunikatorischen Rahmen - der nämlich den Zugang zum extraterrestrischen Wissensraum erklärt -, und zwar am besten basierend auf einem zeitgenössisch anerkannten Wissenschaftsdiskurs (beispielsweise dem Mesmerismus innerhalb des Traumdiskurses). Anders als in der Literatur terrestrischer Fiktion ist hier der Entwurf eines szientifisch anerkannten medialen bzw. kommunikatorischen Milieus unverzichtbar für eine einwandfreie Funktionsweise der Poetik der negierten Fiktionalität. Es braucht somit eine Menge imaginierter Poetologie, um die Fiktionalität des Textes zu eliminieren, eine Poetologie, die sich jedoch selbst als solche nicht zu erkennen gibt. 
Die Vorstellung von extraterrestrischen Räumen in der Literatur um 1800 scheint massgeblich durch mittelalterliche Jenseitsdarstellungen inspiriert zu sein. Erklären lässt sich eine solche Koinzidenz als Konsequenz der Übernahme von Konstituenten, die die antiken Kosmologien eines Platons oder Aristoteles strukturieren (Symmetriesierung und Entmaterialisierung) und nun als Grundlage einer Kartographie des bewohnten Kosmos in der postkopernikanischen Weltall-Fiktion fungieren. Die Kartographie des Kosmos, auf der die Ordnung der bewohnten Plätze basiert, entspricht jedoch dem modernen szientifischen Weltbild. Es werden detaillierte Angaben zu den besuchten Himmelskörpern gemacht, um das damalige Wissen der Astronomen empirisch zu überprüfen. Auch das kopernikanische Weltbild wird kaum hinterfragt. Der Hierarchisierung und Darstellungsweise der bewohnten Räume und ihres Wertes im System des bewohnten Universums liegen jedoch Konstituenten zugrunde, von denen auch die mittelalterliche Kosmologie lebt.

Die Poetik der negierten Fiktionalität — so kann das literarische Verfahren vieler extraterrestrischer Texte um diese Zeit interpretiert werden. Sie entwerfen für sich eine Maske, die das Poetisch-Fiktionale geradezu vertuschen soll (beispielsweise durch die Integration der szientifisch fundierten Facta in die Narration). Die Spezifik der Weltraumliteratur besteht jedoch darin, dass sie einen medialen Rahmen benötigt, in den sich die Erzählung einfügt, um den Status der eigenen Narration epistemisch aufzuwerten: ohne mediale Rechtfertigung der Narration keine epistemische Inszenierung als Weltallexpedition. Der Traum ist nicht nur ein Exempel für ein solches mediales Arrangement, sondern auch ein Fallbeispiel, an dem sich eine fruchtbare Allianz der Poetik und Wissenschaft zeigen lässt. Denn was das Konzept der negierten Fiktionalität anbelangt, muss der mediale Rahmen, der jene Poetik geradezu rechtfertigen soll, szientifisch begründet werden. Es ergibt sich aus dieser Beobachtung, dass ausgerechnet die Wissenschaft (bzw. ihr Verhältnis zum Mediatisierungsinstrument der Erzählung) als ein wichtiges, wenn nicht zentrales Mittel 


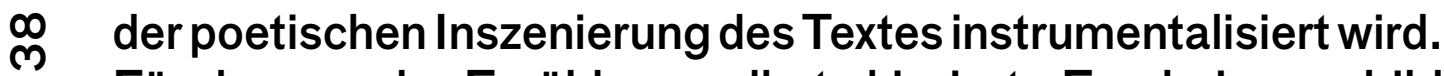
Für das von der Erzählung selbst skizzierte Erscheinungsbild ist es entscheidend, ob die Mediatisierungsmechanismen der Narration wissenschaftlich fundiert sind oder eben nicht. So entwirft die Hypermediatisierung durch die Interferenz der Fiktionalitätsmedien in Keplers Somnium, das heisst durch eine bewusste Entwissenschaftlichung des medialen Rahmens, in den sich die Narration einbettet, ein völlig anderes poetisches Erscheinungsbild des Textes als beispielsweise die szientifische Fundierung jener Mediatisierungsmittel der Narration in den somnambulen Romanen. Die Medien, die die Erzählung innerhalb der Narration mediatisieren, und ihre Beziehung zur Wissenschaft spielen eine entscheidende Rolle für das literarische Autoporträt des Textes. 
1 ARTHURO. LOVEJOY, DIE GROSSE KETTE DER WESEN : GESCHICHTE EINES GEDANKENS, ÜBERS. VON DIETER TURCK, FRANKFURT AM MAIN 1993 [1933], S. 126.

\section{CAMILLE FLAMMARION,} SPAZIERGÄNGE INDER STER-

NENWELT. WISSENSCHAFTLICHE VOLKSBÜCHER FÜR SCHULE UND HAUS, HG. VON FRITZ GANSBERG, ÜBERS. VON ELISABETH MOSENGEL, HAMBURG 1920 S. 26-37. DIE RELEVANZ DER MIKROSKOPIE FÜR DIE DISKUSSION ÜBER DIE WELTENPLURALITÄT WIRD IN ZAHLREICHEN POPULÄRWISSENSCHAFTLICHEN SACHBÜCHERN THEMATISIERT, Z. B. BRUNO H. BÜRGEL, AUS FERNEN WELTEN. EINE VOLKSTÜMLICHE HIMMELSKUNDE. NEUBEARBEITETE UND ERWEITERTE AUSGABE. MIT 280 ABBILDUNGENIM TEXT UND 48 TAFELN, BERLIN 1949 [1910], S. 94; ODER AUCH JOSEPH POHLE, DIE STERNWELTEN UND IHRE BEWOHNER:EINE WISSENSCHAFT LICHE STUDIE ÜBER DIE BEWOHNBARKEIT UND DIE BELEBT HEIT DER HIMMELSKÖRPER NACH DEM NEUESTEN STANDPUNKTE DER WISSENSCHAFTEN, KÖLN 1884-1885, S.428; ZUR BEDEUTUNG DER SPEKTRALANALYSE FÜR DIE ASTRONOMIE VGL. BEISPIELSWEISE KARL KÜPPERS, VERSTÄNDIGUNG ZWISCHENERDE UND MARS, BERLIN 1924, S. 15.

3 TITUS LUCRETIUS CARUS, DE RERUM NATURA: DIE WELT AUS ATOMEN, LATEINISCH UND DEUTSCH,LEIPZIG 1928, S.585.

4 GIORDANOBRUNO, UEBER DAS UNENDLICHE, DAS UNIVERSUM UND DIE WELTEN, ÜBERS. VON CHRISTIANE SCHULTZ, STUTTGART 1994. 5 IMMANUEL KANT, ALLGEMEINE NATURGESCHICHTE UND THEORIE DES HIMMELS, BERLIN 1955 [1755], S. 38. EMANUEL SWEDENBORG, DIE ERDKÖRPER IM WELT ALL UND IHRE BEWOHNER, REVIDIERTE ÜBERS. NACH IMMANUEL TAFEL, ZÜRICH 1997 [1758], S. 17.

JOHANN HEINRICH LAMBERT, COSMOLOGISCHE BRIEFE ÜBER DIE EINRICHTUNG DES WELTBAUES, AUGSBURG 1761, S. 135-7, 147, 158 .

AUCH IMMANUEL

KANT BESCHÄFTIGT SICH IN SEINER ALLGEMEINEN NATURGESCHICHTE UND PHILOSOPHIE DES HIMMELS MIT SYSTEMATISCHEN INTERDEPENDENZEN ZWISCHEN EINZELNEN SON-

NENSYSTEMEN UND DER ÜBERGEORDNETEN STRUKTUR DER MILCHSTRASSE: «DIE GESTALT DES HIMMELS DER FIXSTERNE HAT ALSO KEINE ANDERE URSACHE, ALS EBEN EINE DERGLEICHEN SYSTEMATISCHE VERFASSUNG IM GROSSEN, ALS DER PLANETISCHE WELT BAU IM KLEINEN HAT, INDEM ALLE SONNEN EIN SYSTEM AUSMACHEN, DESSEN ALLGEMEINE BEZIEHUNGSFLÄCHE DIE MILCHSTRASSE IST» (KANT, ALLGEMEINE NATURGESCHICHTE (ANM.5), S. 58).
EMANUEL SWEDEN ORG VERTRITT ALS EINER DER BEKANNTESTEN VERFEC TER DES KARTESIANISCHEN WELTMODELLS EINE ÄHNLICHE ANSICHT. NICHT NUR SOLL SEINES ERACHTENS JEDES SON NENSYSTEM IN EINEM WIRBEL KREISEN, SONDERN AUCH DIE UNZÄHLIGEN WIRBEL JENER SYSTEME SIND WIEDERUM KOMPONENTEN EINER METADIMENSIONALEN ORDNUNGSSTRUKTUR, RICHARD L. GOERWITZ, AUSSERIRDISCHES LEBE IN: SWEDENBORG, ERDKÖRPER IM WELTALL (ANM.6), S. 176-230, HIER S. 217-218.

HANS SCHAVERNOCH, DIE HARMONIE DER SPHÄREN: DIE GESCHICHTE DER IDEE DES WELTENEINKLANGS UND DER SEELENEINSTIMMUNG, FREIBURG ET AL. 1981, S. 37-60 UND S. 68-72.

$9 \quad$ EBD., S. 158.

EMERICH ZEDERBAUER, DIE HARMONIE IM WELTALL, INDER NATUR UND KUNST, WIEN 1917.

\section{DIE SIGNIFIKANZ DER} MONDE ALS MASSSTAB DER GEISTIGEN PERFEKTION VON

LEBEWESEN, DIE JENEN KOSMISCHEN RAUM BEWIRTEN WIRD THEMATISIERT BEIJOSEPH EMIL NÜRNBERGER, $A S T R O N O M I-$ SCHE REISEBERICHTE ODER TOPOGRAPHIE DES HIMMELS UND PLANETARISCHENMETEMPSYCHOSE, DANNHEIMER 1837, S. 72 UND S. 142F.; ODER BEI HANIA SIEBENPFEIFFER, KOMMENTAR, IN: EBERHARD CHRISTIAN KINDERMANN, GESCHWINDE REISE AUF DEM LUFT $=$ SCHIFF NACH DER OBERN WELT, HG. UND MIT EINEM KOMMENTAR VERSEHEN VON HANIA SIEBENPFEIFFER, UNTER DER MITARBEIT VON STEPHANIE TORGE UND KRISTINJUST, WEHRHAHN 2010 S. 77-104, HIER S. 98.

12 KANT, ALLGEMEINE NA TURGESCHICHTE (ANM. 5), S. 81. 13 EIN ÄHNLICHES KONZEPT FINDET MAN SPÄTER IN DEN SCHRIFTEN DES DEUTSCHEN PHYSIKERS ERNST GOTTFRIED FISCHER: «[...] DER STRAHL DER SONNE, OHNE SELBST FEUER ZU SEYN, SETZT BLOSS DEN BELEBENDEN WÄRMESTOFF IN BEWEGUNG; AUF DEM MERKUR WIRD DIESER STOFF VON GRÖBERER, AUF DEM SATURN UND URANUS VON WEIT SUBTILER NATUR SEYN, ALS BEY UNS, SO DASS DER STÄRKERE SONNENSTRAHL AUF DEM MERKUR, DER MILDERE AUF VENUS, ERDE, MARS UND DER SCHWÄCHERE AUF JUPITER,

SATURN UND URANUS, DOCH NUR GLEICHE WIRKUNG DER WÄRME AUF JEDER DIESER WEL TEN HERVORDRINGEN KANN (ERNST GOTTFRIED FISCHER, ALLGEMEINE BETRACHTUNGEN ÜBER DIE KOMETEN: BEY GE

LEGENHEIT DER VERMUTHETEN WIEDERERSCHEINUNG EINES KOMETENIM JAHRE, BERLIN 1789 S.4). DIE STEIGERUNG DES PERFEKTIONSGRADES HÄNGT HIER JEDOCH NICHT NUR VON DER GROBHEIT DES WÄRMESTOFFS AB, SONDERN AUCH VON DER JAHRESDAUER AUF
DEN PLANETEN. DENN DIE «LEBENSDAUER DER BEWOHNER ANDERER WELTEN» STEHT «IN EINEM SCHICKLICHEN VER HÄLTNISS» «MIT DEN UMLAUFSZEITEN DERSELBEN, D. H. MIT IHREN JAHREN UND JAHRESZEITEN». SOMIT LEBEN DIE BEWOHNER DER ÄUSSEREN (VOLLKOMMENEN) PLANETEN NACH UNSERER RECHNUNG "JAHRHUNDERTE», WAS IHNEN WIEDERUM ERMÖGLICHT, «TIEFER IN DIE GEHEIMNISSE "TER NATUR, TIEFER IN DIE KENNTNISS DES WELTBAUES, TIEFER IN DIE ERKENNTNISS IHRER EIGENEN NATUR UND BESTIMMUNG EINZUDRINGEN (EBD., S.6).JENE EVOLU

TIONISTISCHE KOSMOGENESE FINDET DANN IHREN WIDERHALL IN DER MODERNEN ASTRONOMIE EINES PIERRE-SIMON LAPLACES, WILHELM HERSCHELS ODER FRIEDRICH ZÖLL-

NERS UND GILT EIGENTLICH BIS HEUTE ALS GRUNDMODEL FÜR DIE GENESE UNSERES SON NENSYSTEMS.

14 NÜRNBERGER, $A S T R O$ NOMISCHE REISEBERICHTE (ANM.11), S. 213

15 ANONYM, ZAMORODE DER MANN AUS DEM MON-

DE - KEIN BLOSSER ROMAN, BERLIN 1787, S.32. 16 I.P.COULIANO, CLE MENS WILHELM, JENSEITS DIE SER WELT: AUSSERWELTLI CHE REISEN VON GILGAMESCH BIS ALBERT EINSTEIN, MÜNCHEN 1995, S. 251.

\section{JÜRGEN HAMEL, $G E$}

SCHICHTE DER ASTRONOMIE. IN TEXTEN VON HESIOD BIS HUB $B L E, 2$, ÜBERARB. UND ERW. AUFLAGE, ESSEN 2004, S. 19.

18 COULIANO, JENSEITS

DIESER WELT (ANM. 16), S. 247. 19 HAMEL, GESCHICHTE

DER ASTRONOMIE (ANM. 17), S. 91 20 DANTE ALIGHIERI, DIE GÖTTLICHE KOMÖDIE, ÜBERS.

VON HERMANN GMELIN, ANM. VON RUDOLF BAEHR, NACH WORT VON MANFRED HARDT, STUTTGART 2013.

21 JENE KONSTITUENTEN STIFTEN SOWOHL DEM RAUM (HIMMELSKÖRPER) ALS AUCH SEINEN BEWOHNERN EINE IDENTITÄT. EIN BESTIMM TER GRAD DER ENTMATERIALISIERUNG PRÄDESTINIERT EINERSEITS DIE LANDSCHAFT EINES PLANETEN, DER DIESEN GRAD IM SYSTEM ALLER MÖGLICHEN ABSTUFUNGEN VERTRITT, UND ANDERERSEITS DIE PHYSIOLOGISCHE BESCHAFFENHEIT DER PLANETENBEWOHNER.

22 JOHANN GOTTFRIED

HERDER, ÜBER DIE SEELENWAN DERUNG. DREI GESPRÄCHE

IN: DERS., SCHRIFTEN ZUPHILO SOPHIE, LITERATUR, KUNST UND ALTERTUM 1774-1787, FRANK-

FURT A. M. 1994, S. 425-474, HIER S. 451-452

23 THOMAS WRIGHT, $A N$ ORIGINAL THEORY OR NEW HYPOTHESIS OF THE UNIVERSE, 1750: TOGETHER WITH THE FIRST PUBLICATIONOF A THEORY OF THE UNIVERSE, 1734, FACS. [OFTHEED.] 1750 ED., LONDON 1971.

24 CHRISTOPH MARTIN AND, DIE NATUR DER DIN GE IN SECHS BÜCHERN, MIT

EINER VORREDE GEORG FRIED RICH MEIERS, HALL IM MAGDEBURGISCHEN 1752, S. 14. 25 VGL.ETWADIE TU-

GENDHAFTIGKEIT DER EXTRA TERRESTRISCHEN MENSCHEN (EBD., S. 108)

26 «HOCH ÜBER JENER CHWARM, DIE SICH VON IHR ENTFERNEN,/SITZT MIT ENTWÖLKTER STIRN DIE WEISHEIT BEY DEN STERNEN, UND DRINGT MIT FREYEM BLICK, SO HEITER ALS IHR SINN,/ DURCH ALLER WELTEN RAUM ZUM THRON DER GOTTHEIT HIN./EIN NIE VERSIEGTER STROM VON UNVERFÄLSCHTEM LICHTE/UMFLIESST 
31 DR.RUDOLPHIO, DIE JUNGE HELLSEHERIN. NEUE MITTHEILUNGENDER SOMNAM BULE IPHIGENIA STRUDELLA ÜBER DAS JENSEITS, VONIHREN REISENINDEN MOND, DIE SONNE UND DIE STERNE. NEBST EINEM ANHANG VON VORTREFFLICHEN HEILMITTELN, DER ZWEITEN VERMEHRTENAUFLAGE VIERTER ABDRUCK, TÜBINGEN 1867, S.9.

32 PHILIPPINE DEMUTHBÄURLE, REISENINDENMOND, INMEHRERE STERNE UNDINDIE SONNE: GESCHICHTE EINER SOMNAMBÜLE [D. I. PHILIPPINE DEMUTHBÄURLE] IN WEIL HEIM ANDER TECKIM KÖNIGREICHE WÜRTEMBERG INDEN IAHREN 1832 UND 1833. EIN BUCH, IN WELCHEM ALLE ÜBER DAS JENSEITS WICHTIGE AUFSCHLÜS SE FINDEN WERDEN, SCHÄZLER 1834.

33 FRIEDRICH EBNER, NEUE REISEN INDEN MOND, DIE PLANETEN, SONNE UND ANDERE STERNE: AUS DEM LEBEN DER SOMNAMBULLE MARIE W..

VON U........, AUF DEM SCHWARZWALDE: MERKWÜRDIGE ENT HÜLLUNGEN AUS DEM GEISTER REICHE; NEBST EINEM ANHAN GE ERPROPTER HEILMITTEL UND MAGNETO-ELECTRICITÄT ZUR HEILUNG ALLER NERVÖSEN KRANKHEITEN, ULM 1852 34 DR.RUDOLPHIO, DIE JUNGE HELLSEHERIN (ANM.31) S. 107.

35 ANONYM, HIMMEL UND HÖLLE [UM 1090], IN: DENK-

MÄLER DEUTSCHER POESIE UND PROSA AUS DEM 8. - 12.JAHRHUNDERT, BAND 1: TEXTE, HG. VON KARL MÜLLENHOFF UND WILHELM SCHERER, BERLIN 1892, S. 67-73.

36 DEMUTH-BÄURLE, $R E I^{-}$ SENINDENMOND (ANM.32), S. 25.

$37 \quad$ EBD., S. 35

38 EBD., S.67.

39 EBNER, NEUE REISENIN

DENMOND (ANM. 33), S. 157.

40 EBD., S. 190.

41 EBD., S. 197

$42 \quad$ EBD., S. 198.

43 EBD., S. 238

44 EBD., S. 247-248.

45 DR.RUDOLPHIO, DIE

JUNGE HELLSEHERIN (ANM.31), S. 15.

46 DEMUTH-BÄURLE, REISE INDENMOND (ANM.32), S. 15.

$47 \quad$ EBD., S. 19 48 DR.RUDOLPHIO, DIE JUNGE HELLSEHERIN (ANM.31), S. 10.

49 GOERWITZ, AUSSERIRDISCHES LEBEN (ANM.7), S. 179. 50 ANONYM, HIMMEL

UND HÖLLE (ANM. 35), Z. 123-129. 51 AUCHINDEMBE-

RÜHMTEN BUCH KINDERMANNS GESCHWINDE REISE AUF DEM LUFT $=$ SCHIFF NACH DER OBERN $W E L T(1744)$ SIND DIE SPUREN JENER RHETORIK DER DURCHSICHTIGKEIT UND ENTMATERIA LISIERUNG IN DER BESCHREIBUNG DER MARSMONDBEWOHNER SICHTBAR: «DOCH WAR DIESER CÖRPER [MC: KÖRPER DER MARSMONDBEWOHNER] NICHT SO CRUD, WIE [IHN] DIE MENSCHEN AUF DER UN-
TERWELT HABEN, SONDERN VON EINEM FLUIDEN UND DOCH FEST ZUSAMMEN GESETZTEN CRYSTALLINEN WESEN» (KINDERMANN, GESCHWINDE REISE (ANM.11), S.31). 52 ANONYM, HIMMEL UND HÖLLE (ANM.35), Z.43-44. 53 «NUN BIN ICH AN ORT UND STELLE; SO EBEN TRETE ICH IN AN DAS THOR DER STADT, IN DIE ICH HEUTE GEFÜHRT WERDE; ICH KOMME ABER NICHT SOGLEICH DURCH DAS THOR, DER EINGANG GLEICHT EINEM WALLE, UND GLÄNZT WIE GOLD; MEIN FÜHRER ABER SAGT, ES SEY NICHT VON GOLD; NUR DIESE ART DER STEINE GLEICHE DEM GOLDE.

ICH KANN EUCH DIE HERRLICHKEIT UND SCHÖNHEIT DIESER STADT, WELCH ICH JETZT DURCHWANDERE, NICHT GENUG SCHILDERN [...]» (DEMUTH BÄURLE, REISENIN DENMOND (ANM.32), S. 47). «SIE SIND SCHNEEWEISS, DURCHSICHTIG WIE SONNENSTRAHLEN UND REIN UND KLAR WIE DIAMANTEN» (DR. RUDOLPHIO, DIE JUNGE HELLSEHERIN (ANM. 31), S. 20), VGL. AUCH S. 96: «SÎU IST IN GOLDES SCONI. SAMO DAZ DURHLIEHTE GLAS. ALLÎU DURH SCÔUVIG IOH DURHLUTHER *

[ANONYM, HIMMEL UND HÖLLE [ANM. 35], Z.41-44].

54 «ALLES GLEICHT EINE GROSSEN MÄCHTIGEN STADT, ERBAUT VON DURCHSICHTIGEN STOFFEN UND IN FORMEN, WELCHE MIT DEN AUF UNSERER ERDE BEKANNTEN KEINE AEHNLICHKEIT HABEN; ABER ÜBERALL HERRSCHT EINHEIT UND LIEBE, WELCHE IM LOBE DES ALLERHÖCHSTENS ÜBERFLIESST $\gg$ (DR. RUDOL-

PHIO, DIE JUNGE HELLSEHERIN (ANM.31), S.20)

55 PETER JEZLER,HIMMEL HÖLLE, FEGEFEUER. DAS JENSEITS IM MITTELALTER. EINE AUSSTELLUNG DES SCHWEIZERISCHENLANDESMUSEUMS, ZÜRICH 1994, S. 380.

56 JOHANNES KEPLER, WELTHARMONIK, ÜBERS. UND EINGELEITET VON MAX CASPAR, MÜNCHEN 1973, S. 214. 57 DEMUTH-BÄURLE, $R E I$ SENINDENMOND (ANM.32), S.30.

$58 \quad$ EBD .94.

59 EBD., S. 102.

60 EBD., S. 111.

61 EBD., S.111.

62 DEMUTH-BÄURLE, $R E I$ SENINDENMOND (ANM.32),

S. 68.

63 EBD., S.21. EBNER, NEUE REISENIN DENMOND (ANM. 33), S.38. 65 ANONYM, HIMMEL UND HÖLLE (ANM. 35), Z.117. 66 SCHAVERNOCH, DIE HARMONIE DER SPHÄREN (ANM. 8), S.92-93.

67 «DER MONDIST EIN TREUER BEGLEITER DER ERDE VON WELCHER ER IN DER ERDFERNE 54,644, IN DER ERDNÄHE 48,961 MEILEN ANSTEHT. SEINE BAHN UM DIE ERDE IST 400 MAL KÜRZER ALS DIE DER ERDE UM DIE SONNE, UND WIRD VON IHM IN 27 TAGEN, 7 STUNDEN, 43 MINUTEN UND
$11 \frac{112}{2}$ SEKUNDEN ZURÜCKGELEGT. DA ER ABER ZUGLEICH AUCH MIT DER ERDE UM DIE SONNE LAUFT, SO BRAUCHT ER, UM MIT DERSELBEN UND DER SONNE WIEDER IN DIE GLEICHE STELLUNG ZU KOMMEN, ODER VON EINEM NEUMOND BIS ZUM ANDERN 29TAGE, 12 STUNDEN, 44 MINUTEN UND 2910 SEKUNDEN. ER HAT DIE GESTALT EINER REGELMÄSSIGEN KUGEL, MIT EINEM FLÄCHENGEHALT VON 688,635 QUADRATMEILEN. IM GANZENIST ER ETWA 50 MAL KLEINER ALS DIE ERDE (EBNER, NEUE REISENINDEN $M O N D$ (ANM.33), S. 156).

68 EBD., S. 156. 69 DR.RUDOLPHIO, DIE

UNGE HELLSEHERIN (ANM.31) S.IV [VORREDE]

70 EBD., S.I [VORREDE]. 71 JOHANNES KEPLER, $I O H$. KEPPLERIMATHEMATICI OLIM IMPERATORII SOMNIUM, SEU OPUS POSTHUMUM DE ASTRONOMIALUNARI, DIVULGATUM À M. LUDOVICO KEPPLERO FILIO, MEDICINAE CANDIDATO, IMPRESSUM PARTIM SAGANI SILESIORUM, ABSOLUTUM FRANCOFURTI 1634, S. 1; DT. ÜBERSETZUNG: «ALS IM JAIRE 1608 DIE ZWISTIGKEITEN ZWISCHEN DEN BRÜDERN KAISER RUDOLPHUNDERZHERZOG MATTHIAS IHREN HÖHEPUNKT ERREICHT HATTEN UND DEREN HANDLUNGEN VIELFACH AUF BEISPIELE AUS DER BÖHMISCHEN GESCHICHTE ZU RÜCKGEFÜHRT WURDEN, RICHTETE ICH, DURCH DIE ALL GEMEINE NEUGIER BEWOGEN, MEINEN SINN DER BÖHMISCHEN LEGENDE ZU, UND ALS ICH DABEI ZUFÄLLIG AUF DIE GESCHICHTE DER DURCH IHRE MAGISCHE KUNST BERÜHMTEN, HELDENMÜTHIGEN ZAUBEINLIBUSS A STIESS, GESCHAH ES EINES NACHTS, DASS ICH, NACH DER BETRACHTUNG DER STERNE UND DES MONDES FÜR HÖHERES EMPFÄNGLICH GEWORDEN, AUF MEINEM BETTE EINSCHLIEF, UND DA SCHIEN ES MIR, ALS LÄSE ICH IN EINEM AUF DER MESSE ERWORBENEN BUCHE FOLGENDES: ... [HERVORHEBUNG: $\mathrm{MC}$ ]» (JOHANNES KEPLER, TRAUM VONMOND. MIT DEM BILDNISS KEPLERS, DEM FAKSIMILE-TITEL DER ORIGINALAUSGABE, 24 ABBILDUNGEN IM TEXT UND 2 TAFELN, LEIPZIG 1898, S.3).

72 ZU EINER ÄHNLICHEN SCHLUSSFOLGERUNG KOMMT FREDERIQUE AÏT-TOUATI, DIE SICH IM ERSTEN HAUPTKA PITEL IHRER MONOGRAPHIE MIT DER FUNKTION DER FIKTIONALITÄT FÜR DIE ERZEU GUNG WISSENSCHAFTLICHER FACTA AUSEINANDERSETZT. KEPLERS SOMNIUM IST ALS EIN GEDANKENEXPERIMENT ( «THOUGHT-EXPERIMENT») KONZIPIERT, EINE IMAGINÄRE, GETRÄUMTE REISE, WELCHE DIE KOPERNIKANISCHE HYPOTHESE PLAUSIBILISIERT, SIE JEDOCH STRINGENT VON DER REDE DES ERZÄHLERS TRENNT, DER DAS SZIENTIFISCH GESICHERTE WISSEN REFERIERT. DAS GETRÄUMTE SUJET

IST AUF EINE «POETIK DES UNDERS» GESTÜTZT UND IST NICHT IN DIE WISSENSCHAFTLICHE REDE DES ERZÄH LERS INTEGRIERT. ANDERS VER HÄLT ES SICH IN FRANCIS GODWINS ERZÄHLUNG THE MAN IN THE MOON OR A DISCOURSE OF A VOYAGETHITHER, BYDOMINGO GONSALES, THE SPEEDY MES SENGER (1638), IN DER DIE SZIENTIFISCHEN FACTA IN DIE IMAGINIERTE ERZÄHLUNG INTEGRIERT WERDEN, WODURCH DAS FIKTIONALE EPISTEMISCH AUFGEWERTET WIRD. WIR HABEN HIER NICHT MEHR MIT EINER «POETIK DES WUNDERS», SONDERN EINER «POETIK DES MÖGLICHEN" ZU TUN, WEIL DIE FIKTIONALE REDE DURCH IHRE INTEGRATION IN DAS FAKTISCHE EPISTEMISCH AUFGEWERTET WIRD (FREDERIQUE AÏT-TOUA- 